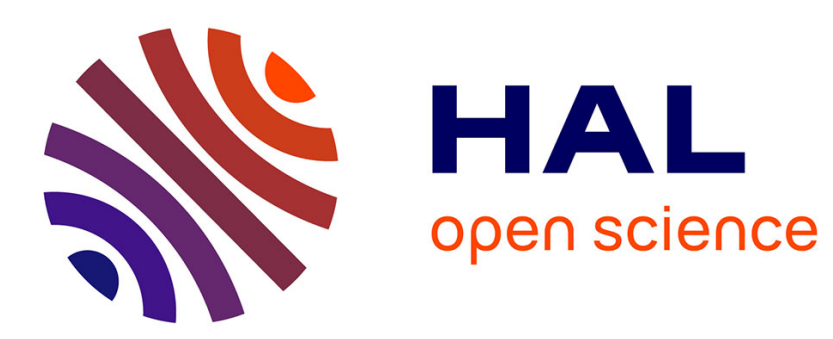

\title{
Travail domestique et travail des enfants, le cas d'Abidjan (Côte-d'Ivoire)
}

Mélanie Jacquemin

\section{To cite this version:}

Mélanie Jacquemin. Travail domestique et travail des enfants, le cas d'Abidjan (Côte-d'Ivoire). Revue Tiers Monde, 2002, Femmes en domesticité: Les domestiques du Sud, au Nord et au Sud, 43 (170), pp.307-326. 10.3406/tiers.2002.1596 . hal-02091300

\section{HAL Id: hal-02091300 https://hal.science/hal-02091300}

Submitted on 5 Apr 2019

HAL is a multi-disciplinary open access archive for the deposit and dissemination of scientific research documents, whether they are published or not. The documents may come from teaching and research institutions in France or abroad, or from public or private research centers.
L'archive ouverte pluridisciplinaire HAL, est destinée au dépôt et à la diffusion de documents scientifiques de niveau recherche, publiés ou non, émanant des établissements d'enseignement et de recherche français ou étrangers, des laboratoires publics ou privés. 


\title{
Travail domestique et travail des enfants, le cas d'Abidjan (Côte- d'Ivoire) \\ Mélanie Jacquemin
}

\begin{abstract}
Mélanie Jacquemin - Domestic work and child labour : the case of Abidjan (Ivory Coast.)

Invisible for a long while or rendered as such, child labour is now in the spotlights due to the present focus on the extreme aspects of child-exploitation. In Ivory Coast, the phenomenon of little servants is undergoing significant changes, combining traditional educational practices through work with more recent wage concerns. Observing the diversity of situations suggests that indiscriminate abolition would be difficult; regulating labour conditions and training of servants may be considered as options to predetermination and better perspectives.
\end{abstract}

\section{Citer ce document / Cite this document :}

Jacquemin Mélanie. Travail domestique et travail des enfants, le cas d'Abidjan (Côte-d'Ivoire). In: Tiers-Monde, tome 43, n¹70, 2002. Femmes en domesticité. Les domestiques du Sud, au Nord et au Sud. pp. 307-326;

doi : $10.3406 /$ tiers.2002.1596

http://www.persee.fr/doc/tiers_1293-8882_2002_num_43_170_1596

Document généré le 25/05/2016 


\title{
TRAVAIL DOMESTIQUE ET TRAVAIL DES ENFANTS, LE CAS D'ABIDJAN (CÔTE-D'IVOIRE)
}

\author{
par Mélanie JACQUEMIN*
}

Longtemps invisible, ou rendu tel, le travail domestique des enfants est en point de mire, au moment où l'urgence focalise l'attention sur les formes extrêmes de l'exploitation des enfants. En Côte-d'Ivoire, le "phénomène des petites bonnes " connaît d'importantes transformations, combinant les pratiques familiales anciennes d'éducation par le travail avec de plus récentes logiques salariales. En observant la diversité des situations, il semble difficile de prôner l'abolition, et plus juste d'orienter la réflexion sur les possibilités de réglementation des conditions de travail et de formation des petites domestiques, pour leur ouvrir des perspectives d'avenir et les aider à sortir du registre de la fatalité.

À Abidjan, si une partie de l'emploi domestique est occupée par des travailleurs adultes (" boys », " grandes bonnes », cuisiniers et cuisinières, nounous, gardiens, jardiniers, chauffeurs de maison), principalement en service dans les fractions dominantes de la société, la majeure partie du travail domestique auquel recourent les fractions populaires comme la petite bourgeoisie est effectuée par des filles de moins de 20 ans. Visible en tous lieux de la ville, l'importance numérique de cette main-d'œuvre jeune (6-20 ans), sous-tendue par d'importants flux migratoires de filles vers la mégapole ivoirienne, apparaît dans les statistiques démographiques : pour Abidjan, la pyramide des âges montre une forte sur-représentation des filles de 10 à 14 ans et de 15 à 19 ans par rapport aux autres groupes d'âge et aux garçons' ${ }^{1}$. Relégué au rang

* Doctorante, Centre d'études africaines, EHess (Paris).

1. Dès 1978 , on relevait à Abidjan un rapport de masculinité (nombre de garçons pour 100 filles) respectivement de 69,1 et de 71,6 pour ces 2 tranches d'âge (cf. P. Antoine et C. Herry, 1982); cette tendance s'est maintenue depuis (cf. Recensement général de la population et de l'habitat (RGPH) 1988 et 1998). 
d'intrigue démographique, cet important déséquilibre a longtemps été négligé dans les recherches: on ne peut l'analyser sans pénétrer l'univers domestique, où le travail est réputé invisible.

En juin 1999, l'OIT a adopté une nouvelle convention internationale visant à éliminer les pires formes de travail des enfants ${ }^{1}$; cette campagne, baptisée 1' "intolérable en point de mire ", cible notamment le travail domestique : les enfants concernés sont considérés comme «les plus vulnérables et les plus exploités de tous les enfants, et aussi [comme] les plus difficiles à protéger ».

En Côte-d'Ivoire, le phénomène des petites domestiques met en jeu l'ensemble de la structure sociale, aussi paraît-il difficile, voire utopique de viser son éradication, du moins à court terme. Centré sur le travail domestique juvénile à Abidjan, ce texte propose de comprendre, par une analyse sociologique des pratiques et des représentations, les logiques d'une telle mise à contribution de fillettes et de jeunes filles dans l'économie domestique. En interrogeant les arrière-plans historique et sociologique des tendances actuelles du " phénomène des petites domestiques ", on repère des modifications diachroniques liées aux changements économiques et sociaux intervenus depuis l'Indépendance (1960), notamment sous l'influence des contextes de grave récession économique qui touchent la Côte-d'Ivoire depuis la décennie $1980^{2}$.

Quelles sont les récentes évolutions de ce phénomène? Les pratiques y sont-elles homogènes ou diversifiées, selon quels déterminants? Dans la vie de ces enfants, que représente l'expérience de travail domestique par rapport à leur devenir?

\section{Pratiques anciennes, secteur informel et pratiques de crise ${ }^{3}$}

Alors que les pratiques anciennes de circulation des enfants (fosterage $)^{4}$ dans les sociétés ouest-africaines en constituent un cadre de référence essentiel ${ }^{5}$, le recours aux services domestiques et mar-

1. La Convention $n^{\circ} 182$ de l'orr a rapidement été ratifiée par un très grand nombre de pays; aucune convention n'avait jusqu'alors connu un tel "succès".

2. Ce travail est issu de recherches effectuées dans le cadre d'un doctorat de sociologie (EHESS, Paris), dont la thèse est en cours de rédaction.

3. Il convient de donner ici au terme de crise un sens pluriel : crise des revenus, "crise de l'école ", crise des modèles d'ascension socio-économique, qui apparaissent comme autant de conséquences sociales de la récession et des politiques d'ajustement structurel engagées depuis 1980.

4. Distinct de l'adoption (cession définitive de l'enfant), le «fosterage " n'implique pas de changement d'identité ni même parfois de localité géographique, les parents conservant une part de droits et de devoirs sur l'enfant concédé, cf. J. Goody, 1969 et S. Lallemand, 1993.

5. Un aspect toutefois peu développé dans ce texte; je renvoie aux études des anthropologues et des ethnologues sur la question, ainsi qu'à mon article dans Journal des Africanistes, 2000. 
chands des enfants est, depuis " la crise », devenu central dans les stratégies familiales de survie. Depuis le début des années 1980 en Côted'Ivoire, les femmes occupent une place grandissante dans l'économie urbaine et dans l'économie des ménages (cf. M. Le Pape, 1997), grâce aux revenus tirés des multiples activités qu'elles exercent, le plus souvent dans le secteur dit informel. Or il est impossible, étant donné les fortes dépenses de temps et d'énergie requises, qu'une femme assure seule l'ensemble du travail domestique et du travail économiquement productif (Cl. Vidal, 1985) : un appoint de main-d'œuvre s'impose, appoint que les fillettes et les jeunes filles non scolarisées, dociles et corvéables, fournissent au meilleur prix.

La plupart des enfants, filles et garçons, participent dès leur jeune âge aux travaux domestiques de leur "maman" (génitrice ou adoptive) ou "tantie" (au sens large), qui ont le rôle d'éducatrices. Aussi celles que j'appelle "petites domestiques» ou «jeunes filles domestiques " (termes génériques) sont des enfants qui exécutent quotidiennement et à plein-temps des activités domestiques, marchandes dans certains cas, sous la tutelle d'un adulte autre que l'un de leurs deux géniteurs.

Je présenterai les trois types de petites domestiques que l'on rencontre aujourd'hui à Abidjan avant d'en décrire les différents modes de placement et de recrutement et de souligner les pratiques plus récentes liées à l'élargissement de ce marché. Le recours à une maind'œuvre juvénile pour effectuer les tâches domestiques ressort bien comme une spécificité du travail domestique en Côte-d'Ivoire, mais il peut aussi être envisagé comme le produit de caractéristiques propres au travail domestique et au travail féminin; l'observation des conditions de vie et de travail des petites bonnes alimentera ensuite cette réflexion, que l'examen des possibilités d'avenir de la "petite domesticité" permettra de prolonger.

CADRE FAMILIAL ET LOGIQUES SALARIALES, LES PETITES BONNES AUJOURD'HUI

De l'enquête ressortent quelques points communs à toutes les petites domestiques: quel que soit leur âge, elles effectuent un minimum de onze heures de travail quotidien, la grande majorité d'entre elles résidant chez leur "employeur"; de fait, logement et nourriture sont constitutifs de la compensation du travail fourni. Enfin, qu'elles soient 
déscolarisées ou qu'elles n'aient jamais "fait les bancs ", leur situation de travail annule toute possibilité de fréquentation scolaire.

Mais l'appellation " (petites) bonnes", communément attribuée aux filles en position de travail domestique, tout comme les expressions génériques que j'utilise, masquent la diversité des situations observées. L'âge, le statut dans l'unité résidentielle où elles travaillent (lié aux modalités de placement et de recrutement), la nature des tâches effectuées, le type de rétribution qu'elles perçoivent, les conditions de travail et le comportement dont elles sont l'objet révèlent cette diversité.

On observe aujourd'hui dans les ménages abidjanais trois types de petites domestiques.

Jeune parente, ou bien proche par l'origine régionale, la "petite nièce"' n'est pas payée en numéraire : elle est logée, nourrie, habillée et assistée en cas de maladie par sa patronne-tutrice qui devra également "faire quelque chose" pour elle quand elle quittera la place (une "valise ": trousseau, pagnes, vaisselle, machine à coudre, petit pécule, etc.), cette dernière obligation étant en principe précédée de transferts (en nature ou en argent) et de services envers les parents de la fille, auxquels la patronne-tutrice peut difficilement se soustraire. La mise au travail de "petites nièces" prolonge une pratique ancienne de circulation des enfants très importante en Afrique de l'Ouest, dans le cadre des réseaux de solidarités (familiales ou autres); ces petites domestiques représentent une sous-catégorie d'enfants fosterés.

Soit l'exemple d'Élisa, 18 ans : originaire d'un village Baoulé (centre du pays), elle y vivait avec ses frères et sa maman qu'elle aidait un peu aux travaux domestiques et champêtres ; elle n'était pas scolarisée et considère qu'elle ne faisait rien. Un jour, Jeanne - petite commerçante abidjanaise originaire du même village - vient trouver la mère d'Élisa et lui demande de lui confier sa fille pour l'aider à s'occuper de son dernier-né. Élisa avait 8 ans. Depuis elle est toujours restée auprès de Jeanne, qu'elle appelle «maman ». De $6 \mathrm{~h}$ à $20 \mathrm{~h}$, elle accomplit les tâches domestiques (ménage, marché, cuisine, vaisselle, lessive, repassage), s'occupe des plus jeunes enfants et gère un petit commerce vivrier (eau et jus glacés, gâteaux secs, bonbons) dans le quartier pour le compte de Jeanne. Cette dernière lui a tout appris. Au fil des ans, elle a "recruté" trois autres "petites nièces" plus jeunes, qui les secondent. Élisa n'a jamais reçu d'argent mais sa "maman » l'a toujours hébergée, nourrie, soignée lors de ses crises de paludisme;

1. À défaut d'expression vernaculaire, j'emploie ce concept à la suite de Marc Le Pape et de Claudine Vidal qui l'utilisent dans leurs recherches. 
elle lui achète des vêtements, des chaussures, de la pommade, paye pour ses tresses et, depuis deux ans, elle a inscrit sa " protégée " à des cours du soir en couture, qu'elle finance. Élisa est revenue trois fois au village rendre visite à sa mère, pour laquelle Jeanne avait acheté des pagnes et du savon. Selon Élisa, on appelle les filles qui font le travail de maison des " servantes ", mais elle ne se considère pas comme telle : «Non! moi, puisque ma tante elle m'a prise, depuis que j'étais petite elle m'a pris, elle ne me paye pas, je suis devenue comme son enfant. " À cette différence près que les enfants de Jeanne sont tous scolarisés et qu'ils aident peu aux tâches domestiques...

Deuxième type, l'enfant louée est salariée dans un ménage avec lequel elle n'a aucun lien de parenté, mais elle est toujours placée chez l'employeur par l'intermédiaire d'un "tuteur" (généralement une femme), le plus souvent venu la chercher au village et qui perçoit et gère son salaire. Elle est habituellement hébergée et nourrie par l'employeur, qui doit également assurer les besoins (vestimentaires, médicaux) de son employée, la tutrice ne laissant en général rien à cet effet $^{1}$.

Les enfants louées ont donc la particularité de recevoir de leur tutrice une rétribution de leur travail sous forme de compensation non monétaire, et seulement au moment de leur retour au village (elles se rapprochent en cela des "petites nièces"), alors que la rémunération de leur travail est monétaire et mensuelle (à la différence des "petites nièces").

Haoua est restée au village (région Nord-Est) avec ses parents jusqu'à l'âge de 10 ans. Elle refusait d'aller à l'école; lorsqu'une tante paternelle est venue la chercher pour l'emmener à Abidjan garder son bébé, elle a tout de suite accepté, encouragée par ses parents. "Petite nièce" pendant deux ans auprès de cette tante, elle voulait ensuite travailler comme bonne : sa tante l'accompagne dans une agence de placement à Adjamé et Haoua trouve une employeuse dans la journée. Cette première patronne la chasse quelques mois plus tard et aujourd'hui Haoua travaille chez une autre femme, que sa tante fré-

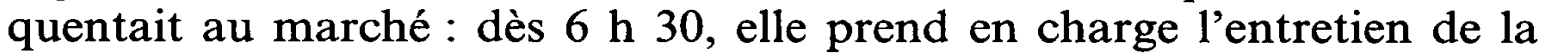
maison, la lessive, elle accompagne sa patronne au marché et l'aide à préparer les repas; l'après-midi, elle remplit et attache des sachets d'eau et de jus glacés qu'elle vend à domicile tout au long de la journée. Depuis qu'elle est servante, Haoua ne voit sa tante paternelle

1. Les enfants louées logent quelquefois chez leur tutrice qui peut ainsi surveiller les plus âgées et parer à d'éventuelles fugues, tout en extorquant encore d'elles des heures de travail à son propre domicile. Lorsque c'est l'enfant elle-même qui remet son salaire mensuel à sa tutrice, elle peut parfois garder une modique somme pour ses « petits besoins" (pommade, savon, chaussures en plastique, etc.). 
qu'une fois par mois : au début, celle-ci passait mensuellement récupérer les $12000 \mathrm{~F} \mathrm{CFA}^{1}$ que sa nièce gagnait pour un mois de labeur dans sa première place ; à présent Haoua se rend chaque mois chez sa tante et lui apporte son salaire mensuel de $10000 \mathrm{~F} \mathrm{CFA}$, desquels elle peut soustraire 1500 à $2000 \mathrm{~F}$ CFA d'argent de poche. Avec les revenus de Haoua, sa tante lui achète des pagnes et de la vaisselle qu'elle garde en prévision de son retour au village, en principe pour le mariage.

En général logée et nourrie chez l'employeur, la petite salariée domestique n'a aucun lien de parenté avec les membres du ménage dans lequel elle travaille ; elle perçoit directement et gère personnellement la rémunération de son travail (salaire monétaire mensuel), dont le montant est en principe fixé au moment de son recrutement. Selon les quartiers, en fonction de l'âge de la fille, du niveau de revenu de l'employeur et quelquefois de la quantité de travail ou de la qualification demandées, les salaires mensuels s'échelonnent de 3000 à $20000 \mathrm{~F}$ CFA, atteignant très exceptionnellement $30000 \mathrm{~F} \mathrm{CFA}$ et plus.

Le terme "salarié" ne correspond pas à son équivalent dans le secteur dit moderne : lorsqu'ils existent, les contrats - bien plus souvent verbaux qu'écrits - demeurent toujours très fragiles, les petites domestiques bénéficiant rarement des dispositions de la législation ivoirienne du travail : elles ne sont jamais déclarées à la CNPS $^{2}$.

Marie, 30 ans, a grandi avec ses parents au village (Nord-Est) où elle a fréquenté l'école jusqu'en $\mathrm{CM} 2$ : au décès de son père, sa mère ne pouvait plus financer sa scolarisation. Quelque temps après, Marie décide de partir à Abidjan travailler pour gagner de quoi s'habiller. À 15 ans (1985), avec l'accord de sa maman, elle part donc à Abidjan avec une tante maternelle qui y vit. Celle-ci accompagne Marie dans une agence de placement à Adjamé où elle trouve très rapidement une patronne, professeur au lycée technique. Malgré l'autorité sévère de cette femme, la jeune fille travaille chez elle pendant cinq ans : "Moi je sais ce que je suis venue chercher, donc j'ai attrapé mon cœur pour travailler." Elle logeait et mangeait chez ses employeurs, entretenait leur maison, préparait les repas, faisait la lessive et s'occupait principalement de leurs deux jeunes enfants, dont l'aîné était scolarisé en maternelle; elle avait obtenu deux week-ends de congé par mois. Marie gagnait $15000 \mathrm{~F}$ CFA par mois avec lesquels elle subvenait à ses besoins (habits, chaussures, savon, pommades, transports); quelques économies lui permettaient d'envoyer à sa

1. Depuis la dévaluation de janvier $1994,100 \mathrm{~F}$ CFA $=1 \mathrm{FF}$, soit $0,15 \epsilon$.

2. Caisse nationale de prévoyance sociale. Il est à noter que même les employés domestiques adultes et qualifiés (boys, cuisiniers, nounous, gardiens, etc.) bénéficient très rarement de cette protection sociale ; entre 1996 et 1999, la CNPS d'Abidjan avait enregistré moins de vingt employés domestiques... 
mère tous les deux mois du poisson, du savon et 5 à $10000 \mathrm{~F}$ CFA. À force de promesses d'augmentation jamais tenues, alors que la quantité de travail ne diminuait pas, Marie a décidé de quitter la place ; sa patronne, en colère, a même repris les vêtements qu'elle lui avait donnés. Après quelques semaines chez sa tante maternelle, Marie retrouve, via l'agence d'Adjamé, un emploi chez une femme malienne : elle s'occupe à plein-temps d'un nourrisson pour $16000 \mathrm{~F}$ CFA mensuels, une autre servante étant chargée du ménage et de la cuisine. Les pleurs de l'enfant l'empêchant de dormir, Marie est partie au bout de six mois. Enceinte de son deuxième enfant, elle a préféré rentrer au village soutenir sa vieille mère; elle y cultive des produits vivriers dont elle fait commerce.

Même lorsque le rapport de travail est monétarisé, la relation de dépendance qui lie les petites domestiques à leur patron(ne) reste forte, hébergement et nourriture participant de la rémunération de leur travail. Toutefois, la perception directe d'un salaire mensuel fixe offre une certaine autonomie aux bonnes salariées, autonomie d'ailleurs liée à l'âge : en effet, il est très rare que le modèle salarial concerne des filles de moins de 13 ans ; lorsque de l'argent vient rétribuer le travail des plus jeunes, elles n'y ont pas véritablement accès, selon le modèle de la location d'enfants. Certaines enfants louées ignorent d'ailleurs le montant, voire l'existence d'un salaire qu'elles ne toucheront jamais; soumises à la double autorité d'une patronne et d'une "tantie» (placeuse et intermédiaire), elles vivent au quotidien des situations de dépendance et d'exploitation liées aux conditions mêmes de leur placement.

DE L'ENTOURAGE AUX AGENCES :

TROUVER UNE BONNE OU UNE PATRONNE

Quel que soit le type de petite domestique retenu, le mode privilégié d'accès à l' "employeur" ou à la petite domestique reste lié à une forme de proximité sociale : familiale, régionale, ethnique, amicale, religieuse, fondée sur une commune appartenance à un quartier, à un métier, à une association... Les formes de mobilisation ou de placement de la main-d'œuvre domestique sont néanmoins très diversifiées : depuis quelques années, la multiplication des agences de placement dans la plupart des quartiers d'Abidjan a encore élargi et modifié ce marché particulier de l'emploi. 
On distingue trois modèles principaux de placement et de recrutement :

Le recrutement direct est le modèle le plus fréquent de captation des "petites nièces"; la future "patronne-tutrice" s'adresse elle-même aux parents de la fille, qui la lui confient en général sans contrepartie immédiate, dans l'espoir (la certitude) de son mieux-être, et par là même du leur. Il arrive que la demande émane des parents, qui souhaitent confier leur fille à une Abidjanaise ; il s'agit donc plutôt d'un placement direct, les conditions étant les mêmes.

Le porte-à-porte des jeunes filles en quête d'une place est un modèle plus récent de placement/recrutement direct, lié au phénomène des "petites bonnes" (salariées) : le plus souvent, la personne intéressée se rend avec sa future employée chez les parents ou tuteurs de la fille pour préciser les termes du "contrat" (montant et destinataire du salaire principalement) et savoir à qui s'adresser en cas de problème. Au détour d'une rue, dans les dédales des marchés, il est fréquent de croiser une jeune fille s'exprimant ainsi : «Bonjour Tantie, pardon je cherche du travail de bonne. "

Le recrutement par personne interposée repose sur un lien plus ou moins étroit entre la demandeuse en quête de main-d'œuvre et une tierce personne (membre de sa famille, du voisinage, amie personnelle ou de son mari, etc.) qui lui trouve une jeune fille. Les petites domestiques ainsi recrutées n'ont aucun lien parental ni amical ni, le plus souvent, ethnique avec leur patronne; leur statut d'enfants louées ou de salariées dépend du contrat passé avec l'intermédiaire du recrutement. Deux figures d'intermédiaires se dessinent :

- certains interviennent seulement pour trouver l'enfant, soit en contrepartie d'une rétribution ponctuelle, en nature ou en argent, prélevée auprès de l'employeur au moment de la remise de l'enfant (peu de cas), soit, plus fréquemment, dans le cadre de relations de réciprocité à plus long terme. Ces intermédiaires ne prennent pas en charge les frais du transport de l'enfant, qui sont financés par les parents ou remboursés par l'employeur à l'arrivée. En fonction notamment de la distance du lieu de résidence des parents de la fille et de la possibilité qu'a l'employeur de s'y rendre, l'intermédiaire peut choisir de demeurer le relais entre l'employeur et les parents de la petite domestique, ou bien il peut demander à l'employeur de prendre directement contact avec les parents de l'enfant recrutée';

1. Il arrive aussi que des jeunes filles en quête d'un emploi domestique sollicitent l'appui d'une personne de leur entourage, intermédiaire occasionnel qui fera coüncider cette demande avec celle d'une employeuse à la recherche d'une bonne, s'il en rencontre une dans son réseau de relations. Des petites domestiques servent quelquefois d'intermédiaires. 
- d'autres, se rapprochant de la figure du loueur d'enfants, restent au contraire seuls médiateurs entre les parents de la fille et son (ses) employeur(s). Ils se présentent comme tuteurs et demandent à percevoir le salaire mensuel de l'enfant, afin de le reverser (très partiellement) à ses parents. En général, les employeurs ne leur donnent rien à la remise de l'enfant. Les "véritables" chefs de réseau, professionnel(le)s inséré(e)s dans des filières internes ou transfrontalières et qui peuvent placer quelques dizaines de filles par trimestre à Abidjan, s'apparentent aux agences informelles de placement. C'est par exemple le cas de femmes originaires de la région de Bondoukou (Nord-Est), surnommées « mamans des bonnes » : elles organisent depuis une trentaine d'années des filières de placement de petites domestiques du Nord-Est vers Abidjan; certaines font même venir de jeunes Ghanéennes, via un réseau Ghana-BondoukouAbidjan.

Dans un contexte de demande croissante en main-d'œuvre bon marché et de diversification des activités rémunératrices, des pourvoyeurs professionnels de petites bonnes se sont installés dans les quartiers d'Abidjan. Depuis une quinzaine d'années, des hommes et des femmes ont créé des agences informelles de placement de «filles de ménage ", élargissant ainsi les possibilités de placement et de recrutement tout en simplifiant la démarche.

Le nombre d'agences de placement s'est considérablement accru à Abidjan ces dernières années : début 2001, plus d'une trentaine avaient pignon sur rue. Le " marché aux bonnes " du quartier Bracodi Bar à Adjamé est la plus ancienne et la plus connue : créée à la fin de la décennie 1980 par quelques personnes ayant repris le "filon" d'un boucher du quartier qui faisait venir des filles de son village (région de Bondoukou) pour satisfaire à la demande de ses clients et amis, il concentre aujourd'hui plusieurs groupes de «promoteurs » qui, de $8 \mathrm{~h}$ à $18 \mathrm{~h}$, accueillent d'un côté les jeunes filles en quête de travail domestique salarié, et de l'autre les femmes - et quelques hommes - à la recherche d'une jeune employée. Les filles s'assoient sur des bancs et attendent une patronne potentielle qui discutera du travail à effectuer et du salaire; si elles ne s'entendent pas, la femme essaie avec une autre fille. Lorsqu'elles ont conclu l'affaire, l'un des placeurs inscrit le nom et l'adresse des deux parties; il délivre un reçu à l'employeuse en échange des $5000 \mathrm{~F}$ CFA de commission qu'elle verse ; en cas de problème, ce reçu lui permettra de revenir chercher une autre fille dans un délai de trente jours. Il est en revanche demandé au nouvel employeur de prendre lui-même contact avec les parents ou le tuteur de la fille 
dans les quarante-huit heures, les placeurs se dégageant de toute responsabilité à son égard. Alors qu'à Bracodi Bar les filles ne versent rien, d'autres agences informelles prélèvent des frais de commission (de 5 à 10000 F CFA) sur les premiers salaires des filles placées. Les jours d'affluence (samedi), au seul coin Bracodi les agents peuvent encadrer une cinquantaine de placements.

Si les "promoteurs" de ces "marchés » présentent leur activité comme une "action sociale destinée à aider [leurs] jeunes sœurs qui ont des problèmes ", les médias ivoiriens les dénoncent régulièrement comme «marchands et marchandes de bonnes». Depuis 1999, le BICE (une ONG suisse) a mis en place un programme de formation et de suivi destiné à organiser, réglementer et harmoniser les pratiques de ces nombreuses agences. Les actions de sensibilisation portent essentiellement sur l'application d'un âge minimal (15 ans), l'interdiction de faire valoir des critères ethniques ou religieux et de retrancher une commission sur le salaire des travailleuses. Des animatrices font des tournées d'information sur les droits et les devoirs des jeunes filles domestiques auprès de celles qui attendent une embauche. De leur côté, les femmes qui recrutent dans ces agences reconnaissent que " les encadreurs font ce qu'ils peuvent", même s'ils ne sont " pas très bien organisés »; elles apprécient surtout de pouvoir choisir leur bonne parmi plusieurs filles, de pouvoir en changer ou encore de pouvoir récupérer leur "caution" si aucune des filles ne fait l'affaire. Ces agences souffrent néanmoins d'une mauvaise réputation, issue des vraies et fausses rumeurs de vols commis par des employées que les agences seraient bien souvent incapables d'identifier au moment des réclamations... En 2001, il n'existait qu'une seule agence de placement officielle et en règle, offrant de véritables garanties aux employeurs et aux employé(e)s; mais ses tarifs restent prohibitifs pour un grand nombre d'Abidjanais.

Apparues au cours de la décennie 1990, les agences ont proposé un modèle inédit de placement des petites domestiques en réponse à des besoins nouveaux, détachés des obligations familiales "traditionnelles" de solidarité et de réciprocité. À côté des formes de mobilisation "hors marché" de la main-d'œuvre domestique juvénile qui prévalaient alors, s'est constitué un type particulier de "marché anonyme du travail ", en pleine expansion. En période de dure récession économique, il est ainsi devenu possible d'ajuster, et ce sans prendre le risque d'ébranler des relations de familiarité toujours utiles, le recrutement et le placement des petites bonnes aux contraintes spécifiques du travail domestique et aux stratégies familiales de survie, fondées sur la multiplication des petites activités rémunératrices. 


\author{
«DISPONIBLES AVANT TOUT »: CONDITIONS DE VIE \\ ET DE TRAVAIL DES PETITES DOMESTIQUES
}

Aux différents types de petites domestiques, corrélés aux modalités de placement et de rémunération, s'ajoute une grande hétérogénéité des conditions de vie et de travail. On observe en réalité un continuum de situations, depuis les relations quasi harmonieuses où chacune des parties trouve son compte, jusqu'aux formes extrêmes d'exploitation, accompagnées de violences verbales, physiques, voire sexuelles.

Si les positions les plus insoutenables sont plutôt bien dénoncées dans les rubriques "faits divers" des média locaux et dans la presse généraliste internationale, il est en revanche très difficile de rencontrer et d'interviewer des petites domestiques victimes de maltraitance dans la place qu'elles occupent. À partir de récits de vie et d'entretiens rétrospectifs, $\mathrm{j}$ 'ai toutefois pu avoir accès à quelques témoignages relatant sévices et privations subis chez de précédents employeurs ${ }^{1}$.

\title{
Des conditions de "serviciabilité $\|^{2}$ variables
}

Le type de rémunération introduit un premier critère de différenciation dans les conditions de vie et de travail, les "petites nièces" et les enfants louées ne disposant d'aucune ressource personnelle pour combler les négligences dont elles peuvent faire l'objet; il arrive ainsi qu'elles n'aient pas même de quoi remplacer leurs sandales en plastique coupées (la paire de " tapettes " coûte $250 \mathrm{~F}$ CFA tout au plus)... On remarque à l'inverse chez de nombreuses jeunes domestiques salariées une coquetterie particulière, qu'elles financent sur leurs maigres revenus. L'argent personnel peut également permettre d'atténuer les insuffisances de la nourriture accordée par l'employeur, notamment en ce qui concerne le petit déjeuner; d'autres domestiques mangent tout à fait à leur faim, parfois mieux qu'au village. L'existence de petits cadeaux (vêtements, argent de poche, savons, etc.) offerts par la

1. Il est également très difficile d'interroger les plus jeunes des petites domestiques, notamment celles qui ont entre 6 et 12 ans... Pour combler ce manque, j'ai procédé par entretiens rétrospectifs, un grand nombre de filles interviewées ayant commencé à travailler avant l'âge de 12 ans.

2. J'emprunte ce concept à Martin Verlet qui désigne ainsi « une condition, personnelle, transitoire, de dépendance et de soumission qui contraint, dans bien des cas, l'enfant travailleur à être pris dans un système d'offre et de demande généralisé de services » $(1996,327)$. 
patronne, surtout au moment des fêtes, révèle aussi la diversité des conditions.

Dans l'ensemble, les petites domestiques dorment peu, elles sont en général les premières levées et les dernières couchées. Les temps de repos sont très variables; ils dépendent de l'espace qui leur est concédé dans le logement de l'employeur : une place dans la chambre des enfants ou dans la pièce principale, un matelas ou une natte, voire un simple pagne sur le sol, plus rarement une pièce à part. Dans les situations les plus difficiles, elles n'ont droit qu'au sol de la cuisine. La présence continue de la patronne ou d'autres membres du ménage joue aussi sur un éventuel temps de repos, que les domestiques s'octroient plus facilement quand elles restent seules à la maison.

Les domestiques salariées bénéficient en principe d'un ou deux jours de congé par mois, quelquefois par quinzaine; elles rentrent alors chez les parents (au sens large) qu'elles ont à Abidjan. La plupart des enfants louées vont une fois par mois chez leur "tutrice", afin de leur remettre leurs gains mensuels ; d'autres tutrices viennent ellesmêmes récupérer le salaire et n'accueillent pas leurs " protégées » chez elles.

Les "petites nièces" «descendent » beaucoup plus rarement, surtout si leurs parents résident loin; elles retournent parfois au village pendant quelques jours ou quelques semaines, en moyenne tous les deux à trois ans.

Enfin, la nature de leurs tâches illustre encore la diversité des conditions des petites bonnes. Pour autant, une typologie du travail domestique juvénile selon ce critère est peu pertinente : elle engage une conception trop restrictive de la catégorie " petite domestique » et occulte certaines caractéristiques fondamentales du travail domestique des enfants.

Primo, la frontière entre travail domestique et travail économiquement productif demeure floue, aussi est-il peu pertinent de caractériser ces jeunes travailleuses en fonction des tâches qu'elles accomplissent. La notion de travail domestique doit être pensée par rapport à l'ensemble des activités ménagères et économiques des ménages urbains, des femmes en particulier. On distingue deux niveaux d'articulation entre la sphère du domestique et celle du domaine marchand, qui ne s'excluent pas nécessairement : d'une part, un processus de délégation des tâches domestiques vers des auxiliaires, comme condition indispensable à l'exercice des activités (scolaires, rémunératrices, professionnelles, etc.) d'autres membres du ménage à l'extérieur de la sphère domestique; d'autre part, un prolongement qui consiste à faire participer ces auxiliaires, en plus de leurs travaux strictement 
ménagers, à des activités de production destinées à la commercialisation, ou à la revente de marchandises (vente ambulante ou sédentaire, dans la rue ou sur les marchés). Ce type de petites activités informelles constitue une extension de la sphère domestique, au cœur de laquelle les petites bonnes sont mobilisées; on ne trouve pas de rupture franche entre le monde domestique et le monde marchand.

En outre, la délimitation des tâches est très élastique, et les horaires des petites domestiques sont extensibles : il n'est pas rare qu'une petite domestique recrutée pour accomplir un certain type de travaux se voie par la suite attribuer des tâches supplémentaires ou - plus exceptionnellement - que lui en soient retirées certaines pour qu'elle se consacre entièrement à d'autres activités.

On observe ainsi un grand éventail de profils de domestiques selon la nature des tâches qu'elles réalisent : par exemple dans un ménage où trois bonnes sont salariées, la plus jeune (11 ans) est exclusivement préposée à la garde du seul enfant (15 mois) de sa patronne, la deuxième (12 ans) est chargée du marché, de la cuisine, de la vaisselle et du ménage, et la troisième ( 15 ans) a été recrutée pour vendre des vêtements avec sa patronne le soir au porte-à-porte ; toutes font la lessive le samedi et peuvent être sollicitées pour effectuer de petites courses dans le quartier ou des services dans la maison. Ailleurs, une seule "petite nièce" (16 ans) s'occupe de la garde et des soins d'un jeune enfant, de la préparation des repas, de la vaisselle, de la lessive et de l'entretien de la maison où elle vit avec trois adultes et deux enfants ; une nièce du chef de ménage, plus âgée, suit une formation de couture mais l'aide pour la cuisine lorsqu'elle n'est pas en cours. Ou encore, une patronne salarie six filles ( 9 à 18 ans) qui se partagent sous ses ordres entre le ménage de la maison et du maquis ${ }^{1}$ installé dans la cour, la surveillance de sa dernière-née, la préparation des différents plats, leur service aux clients et aux membres du ménage, la vaisselle du maquis et de la maison, la lessive, les petites courses d'appoint ; l'une des plus âgées accompagne quelquefois la patronne au marché pour porter les achats.

Même s'il est impossible de généraliser, on remarque toutefois que l'âge de la fille, son lien à la "tantie» ou au chef de ménage, le nombre de résidants dans le ménage, l'activité principale de la « tantie » et bien sûr son "degré de gentillesse" déterminent le type et la quantité de travail extorqué, ainsi que le traitement général de la petite domestique. 
L'âge influence principalement les tâches délicates de la cuisine (pilage du foutou et réussite de la sauce), pour lesquelles une qualification et une expérience minimales sont jugées indispensables. Si les petites filles domestiques participent aussi à la préparation des repas en accomplissant les tâches subalternes, elles le font toujours sous la surveillance d'une cuisinière plus âgée (patronne, autre membre féminin du ménage ou autre bonne) qui assurera la "touche finale »: aucune petite domestique de moins de 14-15 ans ne reçoit l'entière responsabilité de l'alimentation. En revanche la plupart des autres tâches, des soins aux jeunes enfants à la vente d'eau glacée ou d'oranges, en passant par les courses au marché, l'approvisionnement en eau, la lessive ou l'entretien du feu, peuvent être attribuées aux petites domestiques sans tenir compte de leur âge; la tendance serait même d'attribuer la garde des enfants ou la vente ambulante aux plus jeunes...

Par ailleurs, une petite domestique fournira plus de travail dans un ménage de dix personnes que dans un groupe résidentiel de trois... Toutefois, en fonction du nombre de femmes présentes dans le ménage, de leurs activités mais aussi de la valeur qu'elles accordent au travail domestique et des représentations qu'elles ont du rôle de la petite bonne, le labeur de celle-ci peut fortement varier, surtout si plusieurs femmes ou jeunes filles mettent la main à la pâte, ou encore si d'autres domestiques sont employé(e)s au sein du ménage.

Enfin, il est plus probable qu'une petite domestique soit mise à contribution pour les activités économiquement productives (petit commerce, restauration de rue, etc.) si elle est employée par une commerçante ou par une artisane que si sa «tantie » est fonctionnaire ou salariée du secteur moderne.

$\mathrm{Au}$-delà de ces variantes qui influent sur le quotidien, les petites domestiques vivent toutes des situations de dépendance liées à leurs conditions de travail. Pour ces jeunes travailleuses résidantes ${ }^{1}$, le travail est central et façonne toutes les interactions sociales. Bien plus qu'un niveau de qualification, il est attendu d'elles docilité et disponibilité; la relation de travail est dominée par la subordination et l'obéissance à l'adulte employeur, elle développe des formes d'assistance (logement, nourriture, soins, vêtements, argent) qui sont autant de formes de dépendance personnelle, fréquemment doublées de l'autorité d'une tutrice. Les petites domestiques évoluent dans des réseaux sociaux rétrécis, elles ont une marge d'expression réduite et

1. J'ai rencontré très peu de domestiques qui ne résidaient pas chez leur employeur, et aucune d'entre elles n'avait moins de 19 ans. 
accèdent peu à l'information. Beaucoup s'enferment dans la position disqualifiée d'infériorité qui leur est statutairement conférée dans le ménage où elles travaillent et par la société. La stigmatisation vestimentaire et corporelle dont la plupart sont l'objet symbolise une condition basse, vulnérable et dévalorisée, qui rompt avec l'idéal d'un traitement «familial » si souvent déclaré.

\section{"Faire partie de la famille"}

En effet, le plus important pour toutes les petites domestiques - y compris pour celles qui ont été recrutées via les bureaux de placement est d'avoir le sentiment d'être incorporée à la famille employeuse : "Elle me prend comme sa fille, comme sa sœur ». Cet idéal, lié au jeune âge des domestiques, est un discours stéréotypé que tiennent à la fois travailleuses et employeuses malgré la réalité des pratiques, qui s'en éloignent. Même si elles se plaignent de la quantité et de l'extensibilité des tâches, de l'absence de congé, de la faiblesse de la rémunération, les jeunes domestiques désirent être approximativement traitées comme un membre de la famille, en dépit des conditions concrètes qui rappellent leur différence (par rapport aux autres enfants du ménage notamment). Les critères avancés concernent surtout le comportement physique et verbal à leur endroit, le partage d'un savoir-faire minimal, la possibilité de "causer", celle de se reposer et d'accéder à des soins en cas de maladie, la perception effective de la rétribution prévue (cadeaux, salaire) et, dans une moindre mesure, la nourriture. Parallèlement, de nombreuses patronnes conseillent aussi cette conduite «familiale » visà-vis des petites domestiques : "Il faut la prendre comme ton enfant, c'est pas parce que c'est l'enfant des autres que tu dois la frapper, Dieu n'a pas dit ça. Si tu maltraites l'enfant des autres, tu ne sais pas aussi ce que va souffrir ton propre enfant chez quelqu'un d'autre. Non! ta bonne, vraiment, il faut la traiter comme ta fille. Mais il y a d'autres femmes qui sont méchantes, c'est comme ça "'.

La confusion est ainsi entretenue : non seulement les pratiques anciennes de fosterage se combinent aux pratiques salariales plus récentes mais, en outre, les travaux domestiques effectués chez un tiers diffèrent peu de ceux qu'une fille accomplit pour sa propre maman : l'équivoque persiste et des conditions similaires, un traitement " familial ", sont attendus. De plus, dans les ménages où la petite bonne côtoie des enfants de son âge, elle les "remplace" aux tâches domesti-

1. Entretien avec Néné, "patronne-tutrice” de deux “petites nièces", 10 mars 2000. 
ques qui leur incomberaient s'ils n'étaient pas scolarisés, en apprentissage ou fosterés ; cela joue aussi en faveur de ce paradigme familial, qui dissimule le rapport de travail et la valeur du labeur exécuté.

L'idée trompeuse selon laquelle le travail en famille serait sans gravité est ici suggérée ; longtemps admise par les organisations onusiennes, cette conception les empêchait précisément d'apprécier la problématique du travail domestique des enfants à sa juste mesure. Il semblerait en effet qu'une petite sœur ou une nièce directement apparentée soit moins exposée aux négligences, aux violences ou à la surcharge de travail qu'une domestique avec laquelle le lien de parenté est beaucoup plus lâche ou inexistant : la réputation de la tutrice (grande sœur, tante, amie) au sein de sa communauté est en jeu, les enjeux relevant du crédit/discrédit auprès du groupe parental, mais aussi de l'imaginaire de la sorcellerie... On ne peut pour autant considérer en soi le lien familial comme la garantie d'un meilleur traitement ni idéaliser des pratiques traditionnelles, qui peuvent, elles aussi, être oppressives : de nombreux exemples le démontrent à travers le monde. À la suite de l'OIT, l'UNICEF a révisé ses positions au cours des dix dernières années.

QUEL AVENIR POUR LES PETITES DOMESTIQUES?

On distinguera le fait de quitter une place, qui correspond à un renvoi ou à une démission, et celui de sortir de la "petite domesticité", qui marque un tournant dans la vie de ces jeunes filles.

Lorsque les conditions de vie et de travail sont trop dures, les contraintes trop nombreuses et l'oppression trop fortement ressentie, les petites domestiques tentent de quitter la place qu'elles occupent, en général avec l'espoir de trouver ailleurs une meilleure patronne. Mais cette solution ne peut être saisie de la même manière par toutes, elle est soumise à conditions : connaître quelqu'un chez qui aller, pouvoir se déplacer dans la gigantesque Abidjan (se repérer, payer le transport), affronter d'éventuelles critiques de paresse et de faiblesse dans l'entourage familial où elles retournent. Ainsi, la capacité à changer d'employeur varie en fonction de l'âge, de la durée de résidence à Abidjan, du capital scolaire - notamment pour lire des pancartes, manier l'argent, s'exprimer en français - mais aussi du statut de la petite domestique.

En quittant sa patronne-tutrice, une "petite nièce" met en cause la réputation ainsi que les relations familiales (au sens large) ou amicales 
ayant présidé à son placement ; en fuyant son employeur, une enfant louée défie l'autorité de sa tutrice et risque l'humiliation personnelle et familiale d'un retour bredouille au village; tandis qu'en démissionnant, une petite bonne salariée se retrouve plus simplement horsemploi, sans perturber un ensemble de relations sociales. On note d'ailleurs une très forte mobilité de ces petites salariées domestiques.

Du point de vue de la patronne, il est également beaucoup plus facile de "licencier" une bonne (enfant louée ou petite salariée) extérieure au réseau parental ou relationnel, qu'une "petite nièce"; les sautes d'humeur des patronnes aboutissant au renvoi immédiat et définitif de leur bonne contribuent à l'importante rotation des emplois domestiques salariés.

\section{«Quand j'aurai grandi »...}

Pour celles qui ne rejettent pas l'idée de rester dans le service domestique, l'ambition consiste alors à trouver la "bonne patronne " et un salaire plus élevé. Hors des fractions dominantes de la société abidjanaise, elles constatent qu'il est extrêmement rare de rencontrer des domestiques de plus de 20-25 ans. Réalistes, elles savent que les places y sont comptées et surtout qu'il leur manque les qualifications nécessaires, le "bon français ", des savoir-faire techniques et le maintien pour briguer un emploi chez les riches " en haut d'en haut "; faire carrière dans l'emploi domestique prend souvent l'allure d'un rêve inaccessible.

Une fois sorties (ou exclues) de la domesticité, leurs possibilités sont réduites ; elles en connaissent d'ailleurs la liste de façon tout à fait exhaustive. La couture, la coiffure et le petit commerce condensent toutes les perspectives d'accéder à un métier, à un "bon travail ", socialement reconnu. Mais l'argent est indispensable, il leur fait cruellement défaut et toutes celles qui n'ont pu épargner assez sur leurs maigres salaires (c'est-à-dire la grande majorité) ont besoin du soutien, très aléatoire, d'un proche : leur tutrice (cas des "petites nièces" et des enfants louées), un parent, un petit ami ou leur futur mari.

Peu de petites bonnes se réjouissent à l'idée de leur union future : sauf à rêver d'épouser un "bon parti »" elles savent que le mariage les confinera à l'univers domestique (et agricole en milieu rural) et à la débrouille quotidienne. En attendant, elles retournent vivre en famille,

1. Quelques histoires (vraies!), qu'on ne manque jamais de rappeler, entretiennent l'espoir ; la plus spectaculaire : à la fin des années 1970, un ministre répudia sa femme pour épouser sa petite bonne, qui depuis roule en Mercédès... 
en ville ou au village, aident aux travaux domestiques et participent quelquefois à une petite activité rémunératrice ; la plupart ne parviennent pas à rompre le statut de dépendantes. En effet, même celles qui ont bénéficié d'un emploi salarié ne peuvent accéder à l'autonomie résidentielle à la sortie. Certaines sont déjà mères d'un ou deux enfants, qu'elles avaient confiés à un proche parent et auprès desquels elles retournent vivre après leur période de travail.

Le marché du travail domestique juvénile se particularise donc aussi en raison du caractère temporaire des emplois qu'il offre, par définition liés au jeune âge. Aussi est-il difficile de parler de stratégies de mobilité socio-économique pour l'ensemble des petites bonnes ; s'il y a toujours mobilité géographique $-\mathrm{y}$ compris quelquefois au sein d'une même ville -, le plus souvent associée à une immersion dans un milieu social plus favorisé que celui de leurs parents, les expériences transitoires d'emploi domestique ne sont pas la garantie d'une promotion sociale, ni dans ni au-delà du travail domestique. En dépit des connaissances, du savoir-faire pratique, de l'apprentissage de modes de vie urbains qu'elles peuvent acquérir aux côtés de leur(s) "patronne(s)", la majorité de ces jeunes travailleuses au statut dévalorisé se sentent condamnées à ne pouvoir améliorer leur sort ; certaines porteront toute leur vie les stigmates de leur humiliante condition. Peu qualifiées, leurs compétences sont rarement reconnues et les petites domestiques ne voient pas comment échapper au monde des " pauvres » et des exploités, sinon en rêve. Puisqu'elles ne seraient «bonnes à rien ", sinon à tout faire...

Ce texte montre que le recours à une main-d'œuvre très jeune est l'une des spécificités majeures du travail domestique en Côte-d'Ivoire, étroitement corrélé aux conditions socio-économiques du travail féminin. La diversité des situations, la multiplicité des pratiques et la variété des trajectoires des petites domestiques, dans leurs contenus et leurs déterminants, prouvent cependant qu'il est nécessaire de rompre avec les interprétations attribuant l'exploitation de la main-d'œuvre juvénile aux seuls facteurs économiques.

Pour appréhender le "phénomène des petites bonnes " à Abidjan, il est en effet important de se détacher des discours qui envisagent le travail des enfants comme une nouvelle forme d'esclavage née de la crise économique et de la misère des familles. L'utilisation domestique de la main-d'œuvre juvénile, qui certes est parfois proche du "travail servile" d'autrefois, ne peut se comprendre sans faire référence au passé de ces pratiques, notamment aux fondements structurels de la circulation des enfants dans les sociétés traditionnelles d'Afrique noire. 
En observant comment des Ivoiriens des fractions sociales dominées sont passés, au cours des trente dernières années, de logiques domestiques familiales associées à un processus éducatif et de socialisation par le travail, à des logiques davantage salariales d'exploitation des enfants, on peut considérer le phénomène des petites domestiques comme un indicateur du changement social en Afrique noire.

$\hat{A}$ travers la Convention 182 , l'oIT reconnaît pour la première fois un rôle aux mouvements associatifs d'enfants travailleurs ; même si les regroupements de petites domestiques sont difficiles et balbutiants à Abidjan, une évolution est amorcée, appuyée par des ONG. Au programme, des actions visant l'amélioration des conditions de travail, et surtout l'épineux sujet de l'éducation et de la formation de ces jeunes travailleuses, qui sont les ressources humaines de demain. Quelques réussites donnent espoir.

RÉFÉRENCES BIBLIOGRAPHIQUES

Antoine P. et Guillaume A. (1984), Une expression de la solidarité familiale à Abidjan : enfants du couple et enfants confiés, Les familles d'aujourd'hui (Colloque international de Genève, 17-20 septembre 1984), Paris, ORSTOM, $13 \mathrm{p}$.

Antoine P. et Herry C. (1982), Enquête démographique à passages répétés. Agglomération d'Abidjan, Abidjan, ministère du Plan et de l'Industrie de Côte-d'Ivoire, Centre orstom de Petit-Bassam, 491 p.

BICE (Bureau international catholique pour l'enfance), 1998, Les petites bonnes à Abidjan. Travail ou exploitation?, Abidjan, BICE Côte-d'Ivoire, 168 p.

Bonnet M. (1993), Le travail des enfants en Afrique, Revue internationale du travail, 132 (3), p. 411-430.

Diop R. (1987), Secteur " informel " et exploitation du travail des enfants : une étude de deux réseaux pourvoyeurs d'enfants loués à Abidjan, Abidjan, Université d'Abidjan, Département de sociologie, mémoire de maîtrise, (I-XXVII), $99 \mathrm{p}$.

Étienne M. (1979), Maternité sociale, rapports d'adoption et pouvoir des femmes chez les Baoulé (Côte-d'Ivoire), L'Homme, XIX (3-4), p. 63-108.

Franck O. (1985), La mobilité des enfants et l'autosuffisance économique des femmes dans le milieu patriarcal africain, in J. Bisilliat et al. (éd.), Femmes et politiques alimentaires, Paris, Éd. de l'orstom, p. 641-652.

Goody J. (1969), Adoption in cross-cultural perspectives, Comparative Studies in Society and History, 11 (1), p. 55-78.

Guillaume A., Vimard, P. et al. (1997), La circulation des enfants en Côted'Ivoire : solidarité familiale, scolarisation et redistribution de la maind'œuvre, in B. Contamin, H. Memel-Foté (éd.), Le modèle ivoirien en question, Paris, Karthala-ORSTOM, p. 573-590. 
Hazoumé F. (1999), Une vie de bonne, Abidjan/Montréal, CEDA/HMH, 76 p. Jacquemin M. Y. (2000), "Petites nièces" et petites bonnes, le travail des fillettes en milieu urbain de Côte-d'Ivoire, Journal des Africanistes, 70 (1-2), 2000 , p. $105-122$.

Jonckers D. (1997), Les enfants confiés, in M. Pilon, T. Locoh et al., Ménages et familles en Afrique, Paris, CEPED, p. 193-208.

Lallemand S. (1993), La circulation des enfants en société traditionnelle. Prêt, don, échange, Paris, L'Harmattan, $224 \mathrm{p}$.

Le Pape M. (1997), L'énergie sociale à Abidjan, Paris, Karthala, 166 p.

Martin-Koné M. L. (1983), Pain sucré, Abidjan, CEDA-Hatier, Monde Noir Jeunesse, $125 \mathrm{p}$.

Oppong C. (1988), Les femmes africaines : des épouses, des mères et des travailleuses, in D. Tabutin, Population et sociétés en Afrique au sud du Sahara, Paris, L'Harmattan, p. 421-440.

Schlemmer B. (éd., 1996), L'enfant exploité. Oppression, mise au travail et prolétarisation, Paris, Karthala/OrSTOM, $522 \mathrm{p}$.

Veil L. (1998), Problématique du travail et du trafic des enfants domestiques en Afrique de l'Ouest et du Centre, UNICEF, Bureau régional pour l'Afrique de l'Ouest et du Centre, Abidjan, CEPRASs, 78 p.

Verlet M. (1996), Grandir à Nima (Ghana) - dérégulation domestique et mise au travail, in B. Schlemmer (éd.), L'enfant exploité, Paris, Karthala/ORSTOM, p. 311-329.

Vidal C. (1985), L'artisanat féminin et la restauration populaire à Abidjan, in J. Bisilliat et al. (éd.), Femmes et politiques alimentaires, Paris, Ed. de l'ORSTOM, p. 548-556.

Vidal C. (1991), Guerre des sexes à Abidjan. Masculin, Féminin, CFA, in Sociologie des passions (Côte-d'Ivoire, Rwanda), Paris, Karthala, p. 133160.

Vidal C. (1994), La «solidarité africaine »: un mythe à revisiter, Cahiers d'Études africaines, 136, XXXIV-4, p. 687-691. 